\title{
Configurations and singularity of hybrid mechanism
}

\author{
Jian-Guo Luo ${ }^{1, \mathrm{a}, \uparrow}$ and Mao-Yan $\mathrm{He}^{2, \mathrm{~b}}$ \\ ${ }^{I}$ Mechanical and Electrical Engineering Department, \\ North China Institute of Science and Technology, \\ East Surbub of Beijing, China, 101601 \\ ${ }^{2}$ Labour Union, North China Institute of Science and Technology, \\ East Surbub of Beijing, China, 101601 \\ aluojg_1598@126.com, ${ }^{b}$ hmy666@ncist.edu.cn
}

\begin{abstract}
Methods to analysis the DOF(degree of freedom) and singularity of pure serial and pure parallel manipulator, which cannot be used to the hybrid manipulator directly, three type of practical topological units and one type of virtual topological unit adopted, Synthesis method of topological dimensionality of the spatial mobility of each topological unit integrated together, architectures of hybrid mechanism discussed, also the formula and process of its topological dimensionality of spatial mobility presented, singularity analysis of hybrid mechanism can be carried out based on the forgoing results.
\end{abstract}

Keywords: Hybrid mechanism; Topological unit; Dimensionality; Synthesis; Singularity

\section{Introduction}

Serial robots and parallel robots are the basic class of robots, serial robots has characteristics of a large working space, large accumulated error on the end of executor, low stiffness, small load carrying capacity, simple solution in forward kinematics and complex solution in inverse kinematics. While parallel robot has characteristics of a small working space, small accumulated error on the end of executor, high stiffness, large load carrying capacity, complex solution in forward kinematics and simple solution in inverse kinematics. Zhou [1] making use the differential method to get the Jacobi matrix of the parallel mechanism, and the singularity of the parallel mechanism is analyzed by using the singularity analysis method of Gosselin and numerical analysis method. Chen [2] proposed the framework of singularity fault tolerant error correction strategy of parallel mechanism with redundant actuation ased on the singular classification of Gosselin. Ji [3] study the different configuration singularity, and puts forward the measures to avoid the initial and global singularity based on the Jacobian matrix of the mechanism. Chen [4] analysis the condition of singularity by using the Jacobian matrix, and specific method is given to avoid the singularity of parallel mechanism. Lu [5] established the kinematics equation of the robot with 
D-H method, the position inverse solution and Jacobi matrix is obtained by the analysis, condition equation of singularity position of the robot found then. $\mathrm{Du}[6]$ use variable substitution simplify the analytical expressions of the singularity trajectory, three dimension visualization description of singularity trajectory of configuration presented by means of the computer simulation. This paper presents a new approach for the analysis on configuration and DOF and singularity judgment based on the adoption of topological unit of kinematic pair and branch chain and group of branch chains of same affiliation and virtual component, combined with the synthesis of topological dimensionality of each topological unit.

\section{Topological Dimensionality of Hybrid Mechanism}

Each branch of parallel mechanism consists of a a series of kinematic pair units, which fixed on a basis and connected to a common platform component, diagram and topological map of 6TPT just as Figure 1 show. Elements of serial branch of parallel mechanism and serial elements must be included in hybrid mechanism, the necessary and sufficient condition of hybrid mechanism, namely multi topological units of serial branch chains built in parallel and connected with topological units of kinematic pair must be included. $L_{S}, L_{P}$ represent the topological unit of serial kinematic pair and topological unit of group of branch chains of same affiliation, which defined as basic topological unit of series $\mathrm{C}$ and series $\mathrm{B}$ respectively, $\mathrm{C}$ and $\mathrm{B}$ both are the basic topological unit, $L_{S}-L_{P}$ or $L_{P}-L_{S}$ must be exist in the composition of topological map of hybrid mechanism, the correctness of this necessary and sufficient condition can be easily proven by reductio ad absurdum.
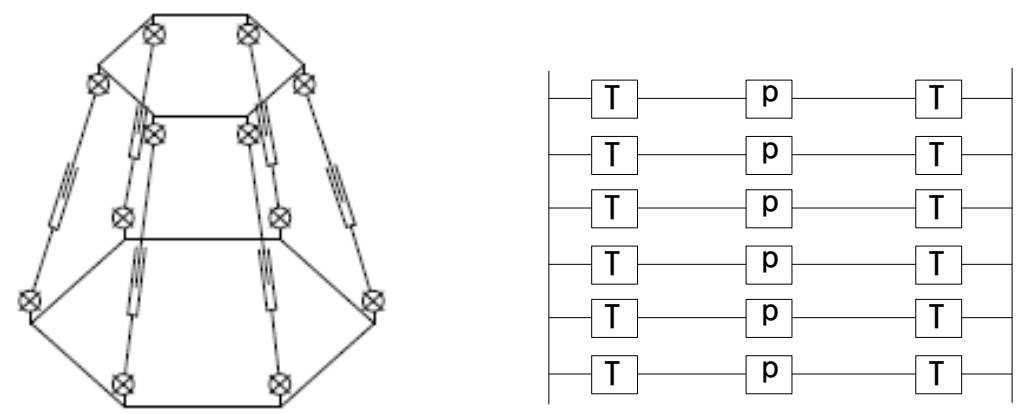

(a) Diagram of 6TPT

(b) Topological structure of 6TPT

Figure 1: 6TPT and its topological map 
Table 1: Topological structure and numbers of configurations of common hybrid mechanism

\begin{tabular}{|c|c|c|c|c|}
\hline \multicolumn{3}{|c|}{$\begin{array}{l}\text { Krmbers of basic } \\
\text { topological unit }\end{array}$} & \multirow{2}{*}{$\begin{array}{l}\begin{array}{l}\text { Topological streucture of serial-parallel. } \\
\text { mechaniom. }\end{array} \\
\mathrm{C}-\mathrm{B}, \mathrm{B}-\mathrm{C} \\
\end{array}$} & \multirow{2}{*}{$\begin{array}{l}\text { Khmbers of } \\
\text { configuration } \\
c_{2}\end{array}$} \\
\hline 2 & $1 \mathrm{c}$ & 1B & & \\
\hline \multirow[t]{2}{*}{3} & $1 \mathrm{C}$ & $2 \mathrm{~B}$ & C-B-B,B-C-B,B-B-C & \multirow{2}{*}{$c_{3}^{1}+c_{3}^{2}$} \\
\hline & $2 \mathrm{C}$ & 1B & $C-C-B, C-B-C, B-C-C$ & \\
\hline ! & ! & $!$ & $!$ & $!$ \\
\hline \multirow{4}{*}{$n$} & $1 \mathrm{C}$ & $n-1 \mathrm{~B}$ & $\mathrm{C}-\mathrm{B}-\mathrm{B} \cdot \mathrm{B}, \mathrm{B}-\mathrm{C}-\mathrm{B} \cdot \mathrm{B} ; \cdot ; \mathrm{B}-\mathrm{B} \cdot \mathrm{B}-\mathrm{C}$ & \multirow{4}{*}{$\sum_{i=1}^{n-1} c_{n}^{i}$} \\
\hline & $2 \mathrm{C}$ & $n-2 B$ & $\mathrm{C}-\mathrm{C}-\mathrm{B} \cdot \mathrm{B}, \mathrm{C}-\mathrm{B}-\mathrm{C}-\mathrm{B} \cdot \mathrm{B} ; ; \mathrm{B}-\mathrm{B} \cdot \mathrm{B}-\mathrm{C}-\mathrm{C}$ & \\
\hline & ! & $!$ & $!$ & \\
\hline & $\mathrm{n}-1 \mathrm{C}$ & 1B & $\mathrm{B}-\mathrm{C}-\mathrm{C} \cdot \mathrm{C}, \mathrm{C}-\mathrm{B}-\mathrm{C} \cdot \mathrm{C} ; \cdot \mathrm{C}-\mathrm{C} \cdot \mathrm{C}-\mathrm{B}$ & \\
\hline
\end{tabular}

In order to found the DOF of common hybrid mechanism under normal condition (non-singularity) based on the spatial moving capability of hybrid mechanism, translation capability $\operatorname{trans}\left(n_{1}\right), \operatorname{trans}\left(n_{2}\right), \cdots, \operatorname{trans}\left(n_{t}\right)$ along the axis $n_{1}, n_{2}, \cdots, n_{t}$ and rotation capability $\operatorname{rot}\left(n_{i}\right), \operatorname{rot}\left(n_{i+1}\right), \cdots, \operatorname{rot}\left(n_{j}\right)$ of $C_{i} / B_{j}$ around the axis $n_{i}, n_{i+1}, \cdots, n_{j}$ defined as the topological dimensionality of basic units $M_{C} / M_{B}$, namely $M_{C} / M_{B}=\left\{\operatorname{trans}\left(n_{1}\right) \cup \operatorname{trans}\left(n_{2}\right) \cup \cdots \cup \operatorname{trans}\left(n_{t}\right) \cup \operatorname{rot}\left(n_{i}\right) \cup \operatorname{rot}\left(n_{i+1}\right) \cup \cdots \cup \operatorname{rot}\left(n_{j}\right)\right\}$, $n_{i}, n_{i+1}, \cdots, n_{j}$ and $n_{1}, n_{2}, \cdots, n_{t}$ can be the same, different, part same and part different. $M_{C}$ can also be named as the topological dimensionality of spatial moving capability of kinematic pair(simplified as topological dimensionality), $M_{B}$ can also be named as the topological dimensionality of spatial moving capability of group of branch chains of the same affiliation. Branch chain consists of more than one kinematic pair in series, $M_{B}$ is the result of intersection after union of topological dimensionality of multi kinematic pairs, the process is defined as the synthesis of topological dimensionality. The same direction of axis, union or intersection take any one is allowed, different direction of axis, all should be included in union and the intersection will not exist. following formula achieved, in expression (1) and (2), $M_{c}^{x}$ is the topological dimensionality of branch chain $x, M_{C}^{x-u}$ is the topological dimensionality of 
kinematic pair $u$ on branch chain $x, y$ is the number of kinematic pairs on branch chain $x, z$ is the number of branch chain in group of branch chains of the same affiliation.

$$
\begin{aligned}
& M_{C}^{x}=\cup_{u=1}^{y} M_{C}^{x-u}, \\
& M_{B}=\overbrace{x=1}^{z} M_{C}^{x},
\end{aligned}
$$

The topological structure of common hybrid mechanism defined as topology following,

$$
\begin{gathered}
B_{1}\left|B_{2}\right| \cdots\left|B_{f}-C_{f+1}-C_{f+2}-\cdots C_{i}-B_{i+1}\right| B_{i+2}|\cdots| B_{j}-C_{j+1}- \\
C_{j+2}-\cdots-C_{k}-B_{k+1}\left|B_{k+2}\right| \cdots \mid B_{s}-C_{s+1}-C_{s+2}-\cdots-C_{t}
\end{gathered}
$$

In expression (3), $f \leq i \leq j<k \leq s \leq t$, and $f, i, j, k, s, t$ is any natural number, signs - and | represent serial relationship and parallel relationship respectively, intersection of union of basic topological unit of $\mathrm{B}$ series and basic topological unit of $C$ series, expression $\left(B_{1}\right), B_{2}, \cdots, B_{f}$ atter three cycles as before, new structure as the result in parallel of $B_{1}, B_{2}, \cdots, B_{f}$ (branch chains of the same affiliation) defined as the virtug component $V C_{1}$, new structure as the result in serial of $C_{f+1}, C_{f+2}, \cdots, C_{i}$ (serial kinematic pairs) defined as the yirtual component $V C_{2}$, the remaining four virtual components defined as $V C_{3}, V C_{4}, V C_{5}, V C_{6}$ in turn, the topological structure of mechanism can be transferred into following,

$$
V C_{1}-V C_{2}-\cdots-V C_{6},
$$

By making use of the formula given in equation (1) and (2), topological dimensionality of this hybrid mechanism can be achieved. First to obtain the topological dimensionality of basic topological units of $\mathrm{C}$ series and $\mathrm{B}$ series, take the virtual component in expression (4) as one topological unit of virtual kinematic pair, result will be obtained as following,

$$
\begin{gathered}
M_{V C_{1}}=\underset{x=1}{\cap} M_{B_{x}} ; M_{V C_{3}}=\underset{y=i+1}{\cap} M_{B_{y}} ; M_{V C_{5}}=\overbrace{z=k+1}^{s} M_{B_{z}}, \\
M_{V C_{2}}=\bigcup_{u=f+1}^{i} M_{C_{u}} ; M_{V C_{4}}=\bigcup_{v=j+1}^{k} M_{C_{v}} ; M_{V C_{6}}=\bigcup_{w=s+1}^{t} M_{C_{w}},
\end{gathered}
$$




$$
M=\bigcup_{q=1}^{6} M_{V C_{q}}
$$

Virtual components $V C_{1}, V C_{2}, \cdots, V C_{6}$ belong to the same level, there is no relationship of inclusion and cross between them, at least one topological dimensionality contained in each virtual component, that is $M_{V C_{q}} \neq \phi$. The DOF of mechanism can be obtained from the number of dimensionality $\mathrm{M}$ of hybrid mechanism.

\subsection{Judgment of Singularity of Hybrid Mechanism}

Topological dimensionality of mechanism can be obtained from the method of synthesis of topological dimensionality, and the general expression of topological dimensionality of spatial moving capability of mechanism can be deduced as following,

$$
M=\left\{\begin{array}{r}
\operatorname{trans}\left(n_{1}\right) \cup \operatorname{trans}\left(n_{2}\right) \cup \cdots \cup \operatorname{trans}\left(n_{t}\right) \\
\cup \operatorname{rot}\left(n_{i}\right) \cup \operatorname{rot}\left(n_{i+1}\right) \cup \cdots \cup \operatorname{rot}\left(n_{j}\right)
\end{array}\right\},
$$

In expression (8), $n_{i}, n_{i+1}, \cdots, n_{j}$ and $n_{1}, n_{2}, \cdots, n_{t}$ can be the same, different, part same and part different. When singularity occurs in the mechanism, parallel or overlap of two axes at least will occurs between axes $n_{i}, n_{i+1}, \cdots, n_{j}$ or between axes $n_{1}, n_{2}, \cdots, n_{t}$ or between axes $n_{i}, n_{i+1}, \cdots, n_{j}$ and axes $n_{1}, n_{2}, \cdots, n_{t}$, which will decrease the number of topological dimensionality of hybrid mechanism, overlap or disappear of at least one topological unit will occur in the topological unit of kinematic pairs and topological unit of branch chains and topological unit of group of branch chains of the same affiliation.

Collection consist of $n_{i}, n_{i+1}, \cdots, n_{j}$ defined as $H_{t}$, Collection consist of $n_{1}, n_{2}, \cdots, n_{t}$ defined as $H_{r}$, axis $n_{p}$ and axis $n_{m}$ in parallel or overlap of $H_{t}$ or $H_{r}$, if $n_{p}$ is the axis included in the spatial moving capability of basic topological unit of $\mathrm{C}$ series, and if $n_{m}$ is the axis included in the spatial moving capability of basic topological unit of $\mathrm{B}$ series, and there will no inclusion relationship between $\mathrm{B}$ and $\mathrm{C}$, and the topological unit of $\mathrm{B}$ is not the element of topological unit of B of higher level( suggest there will at least two topological unit of B series included in the mechanism), the synthesis of topological dimensionality occurs in the same level topological unit, this singularity defined as large singularity, the reduced volume of spatial moving capability of hybrid 
mechanism in this condition indicated as $W_{s}^{u n i}$. If $n_{p}$ and $n_{m}$ belong to the different level topological units respectively, singularity in this condition defined as small singularity, the reduced volume of spatial moving capability of hybrid mechanism in this condition indicated as $W_{s}^{\text {disuni }}$. There will $W_{s}^{\text {disuni }} \in W_{s}^{\text {uni }}$ exists when these two kinds of singularity occur in the same mechanism.

\subsection{Example Analysis}

Take the hybrid mechanism for example, which as show in Fig. 2, B1 denote the 3-RPR parallel mechanism, $\mathrm{C} 1$ denote the translation joint along the axis $\mathrm{Oz}, \mathrm{C} 2$ denote the translation joint along the axis Oy, make use of the method described from the above, we can found its degree of freedom and its singularity.

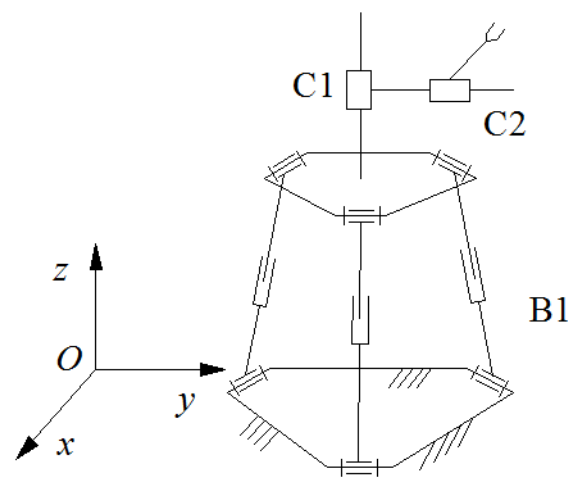

Figure 2: 3-RPR-PP hybrid mechanism

Firstly, the topological structure of the parallel part B1 of hybrid mechanism mechanism can be transferred into virtual component $V C_{1}$, which equivalent to a translation pair $\mathrm{P}$ along the axis $\mathrm{Oz}$.

Secondly, according to expression (1) and (2) and (8), we get the topological dimensionality of the whole mechanism include B1 and $\mathrm{C} 1$ and $\mathrm{C} 2$,

$$
M=M_{B} \cup M_{C}=\{\operatorname{trans}(O z) \cup \operatorname{trans}(O z) \cup \operatorname{trans}(O y)\},
$$

Thirdly, from the expression (9), we can know the dimensionality of this hybrid mechanism is 2, namely the DOF of this mechanism is 2 .

Finally, the reduced volume of spatial moving capability of part B1 in this condition indicated $W_{B}=\{\operatorname{trans}(O x) \cup \operatorname{trans}(O y) \cup \operatorname{rot}(O x) \cup \operatorname{rot}(O y) \operatorname{rot}(O z)\}, \quad$ the reduced volume of spatial moving capability of part $\mathrm{C} 1$ and $\mathrm{C} 2$ in this condition 
indicated as $W_{C}=\{\operatorname{trans}(O x) \cup \operatorname{rot}(O x) \cup \operatorname{rot}(O y) \operatorname{rot}(O z)\}$, thus we
found the result $W_{C} \in W_{B}, W_{C} \quad$ is the large singularity,
$W_{B}-W_{C}=\{\operatorname{trans}(O y)\}$ is the small singularity.

\section{Conclusion}

New approach to analysis the DOF and singularity proposed by making use of the theory on topological unit of kinematic pair and branch chain, as well as group of branch chains of same affiliation and virtual component etc. Included, through the synthesis of topological dimensionality of each topological unit, Formula and process for the topological dimensionality of spatial moving capability of commonly hybrid mechanism obtained with given number of topological unit of $\mathrm{C}$ series and B series, singularity can also be judged from the this results, the relationship between singular working space found as well. Example analysis carried out to identify the validity of this method, which will play great importance in relative research in hybrid mechanism.

\section{Acknowledgement}

This research was financially supported by the HeBei Province Science and Technology Foundation (No.Z2015072, No.15275508) and NCIST Foundation (No. 3142015023).

\section{References}

[1] P. Zhou, P. X. Fu: submitted to Journal of China Mechanical Engineering (2014)

[2] S. Chen, S. W. Fan: submitted to Journal of China Mechanical Engineering (2011)

[3] Y. Ji, H. Z. Liu, D. N. Ding: submitted to Journal of Mechanical science and technology (2012)

[4] B. F. Chen, W. S. Ding, L. P. Ding, et al. : submitted to Journal of South China University of Technology( 2014)

[5] N. Lu, X. H. Zhao: submitted to Journal of Tianjin University of Technology (2013)

[6] Q. Q. Du, Z. H. Guo, Q. J. Guo: submitted to Journal of machine and hydraulic (2015) 